\title{
The Utilization of Wechat for Middle School Education
}

\author{
Guiyuan $\mathrm{Hu}^{1, \mathrm{a}}$ and Chunfen $\mathrm{Bu}^{2, \mathrm{~b}^{*}}$ \\ ${ }^{1}$ The WenLin school of Kunming, department of Chinese education, Kunming Yunnan \\ ${ }^{2}$ Department of physical science and technology, Kunming University, Kunming, Yunnan \\ a1275200871@qq.com \\ b18459423@qq.com \\ *The Corresponding Author
}

Keywords: Middle school education; WeChat; Similarity measure; Data security

\begin{abstract}
WeChat has played a great role in promoting the field of middle school education. In this paper, we aims to design and implement the instruction system based on WeChat. In this paper, this system is discussed in details including its design and implement, at last, its executing efficiency is showed. The exact experimental results indicate that the design objective of this paper is achieved.
\end{abstract}

\section{Introduction}

Modern Internet has become a common way of communication, combined with the mobile Internet, the online education has also been widely recognized, mobile learning as a rapid development of online education is developed rapidly. At the same time, the research of mobile learning in Colleges and universities is in full swing, but the mobile learning platform for public security colleges and universities is less. This work for the public security institutions anti drug education features, with the help of WeChat public platform to build anti drug education and publicity platform for the students to learn anti drug courses in Yunnan Poice College to provide learning environment. This work aims to develop anti drug publicity and education work of media applications, further strengthen the information-based degree of the anti drug publicity and education work, to expand students' learning channels of anti drug education knowledge, innovative drug control publicity and education carrier, deepen the teaching effect of drug.

The reason that we build this platform with the WeChat public platform is that WeChat has played a great role in promoting the field of middle school education. First of all, WeChat's biggest advantage is the establishment of an interactive platform, a business to individuals, businesses on the collective, here, the enterprise is to refer to the use of the platform to disseminate information. In our here is the interaction between the school and students platform, it has a large number of users to use. Micro channel interactive is quite large and teaching how to teach well is often established in which both sides interaction based on. In addition, the micro channel can also meet the diversity of teaching. For example, we use text, pictures, video, spread, and the students have what problem can be in the micro channel to communicate, the communication is not limited in the student and teacher, student and student exchanges. It is like a big community, can be better.

In predecessors' work, the theory and the applications of the construction of instruction system based on WeChat is discussed. In[1-3], the description length which is used to illustrate the performance of statistic data is discussed in details. In [4,5], the calculation method to obtain the description length is given, which is the basis of of our work. On the basis of these constructive works, with the possibility of the instruction system construction discussed in [6,7]. We aims to design and implement the instruction system based on WeChat. In this paper, this system is discussed in details including its design and implement, at last, its executing efficiency is showed. 


\section{Design}

In this section, the high education with the course based on WeChat is discussed.

Firstly, as the example, the platform is developed in order to build the corresponding anti drug publicity and anti drug education platform with WeChat public platform for our students to learn the relevant anti drug courses and drug education courses.

The objective of our design is listed as follows:

a. High flexibility, with a response type, support most of the terminal mobile devices, update simple.

b. Interactivity is strong, the user can communicate with the developer and teacher at all times.

The development of WeChat is based on PHP and the design processing is similar with other web applications. In this paper, the design processing is not given under the limit of pages. In next section, the implement of our instruction system based on WeChat is given.

\section{Implement}

Firstly, Download WeChat in the mobile terminal and the structure is given as shown in figure 1,

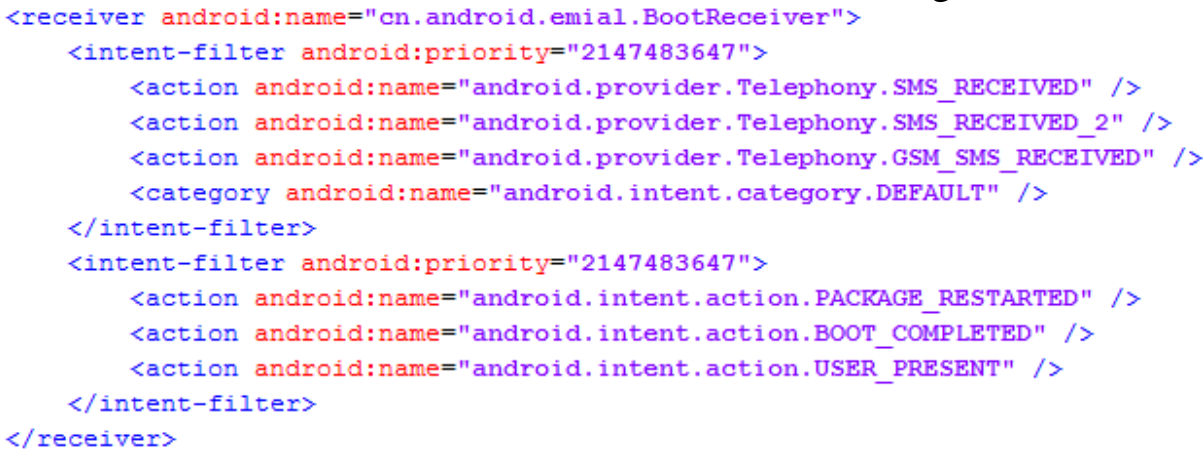

Figure 1. The basic structure of the system based on this paper

Then the implements of this design is based on AndRoid, the exact content discussed are given as Figure 2,

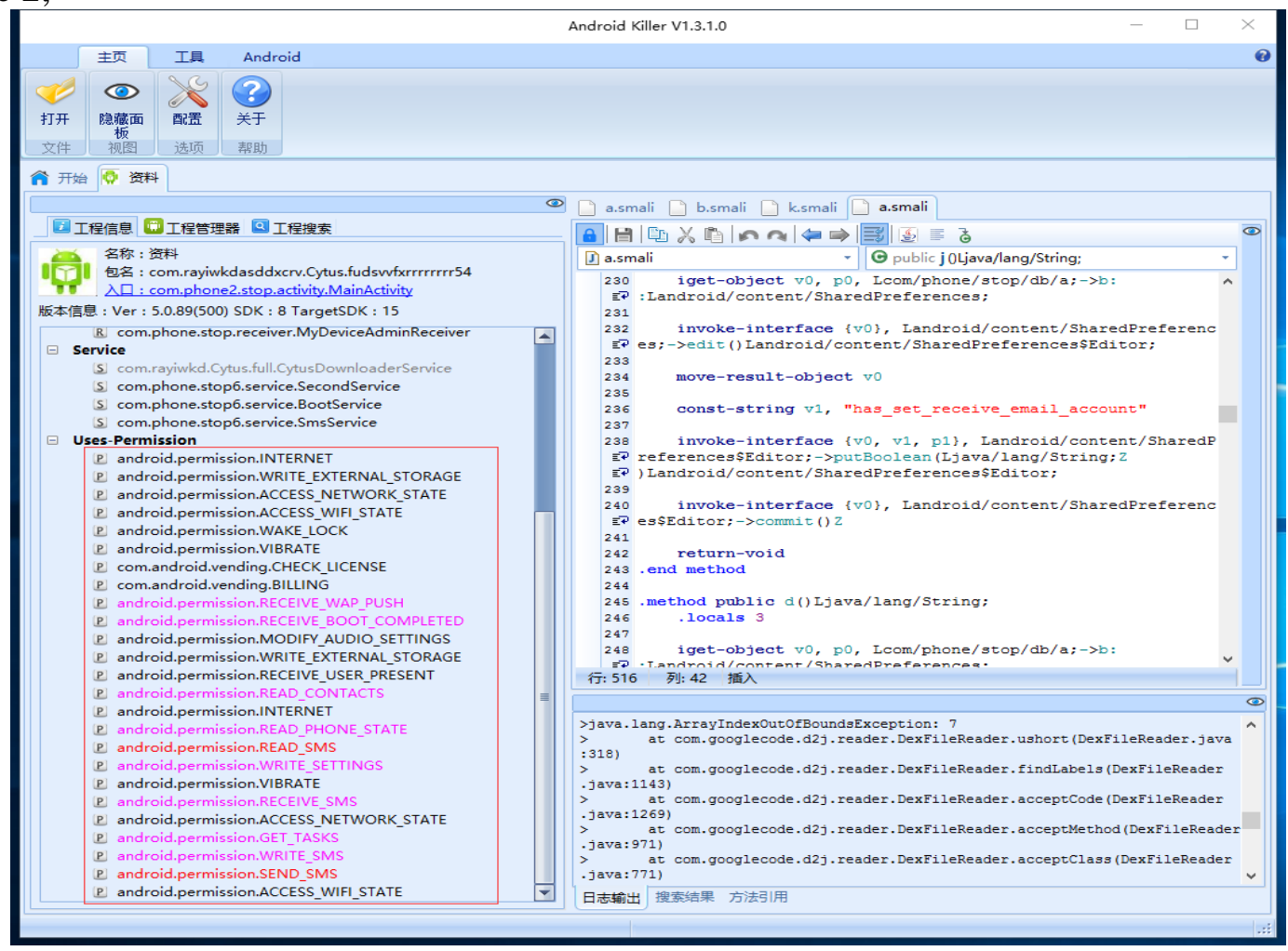

Figure 2. The implement of the corresponding design. 
Try E-mail via E-mail account, it occurred when have time, account report through multilateral or background detection have malicious behavior, has been frozen, unable to log in, if you want to know the mailbox receive email can only be obtained by other methods.

If the above analysis can be completed in a timely manner, it will provide an effective clue to the investigation of the case.

Then new increment in the second quarter of 2016 mobile malware infection and statistics (figure 2,), the new sample size increasing trend, in June 1.748 million, most contracted quantity is on the decline, reached the lowest in June, for 19.33 million times.
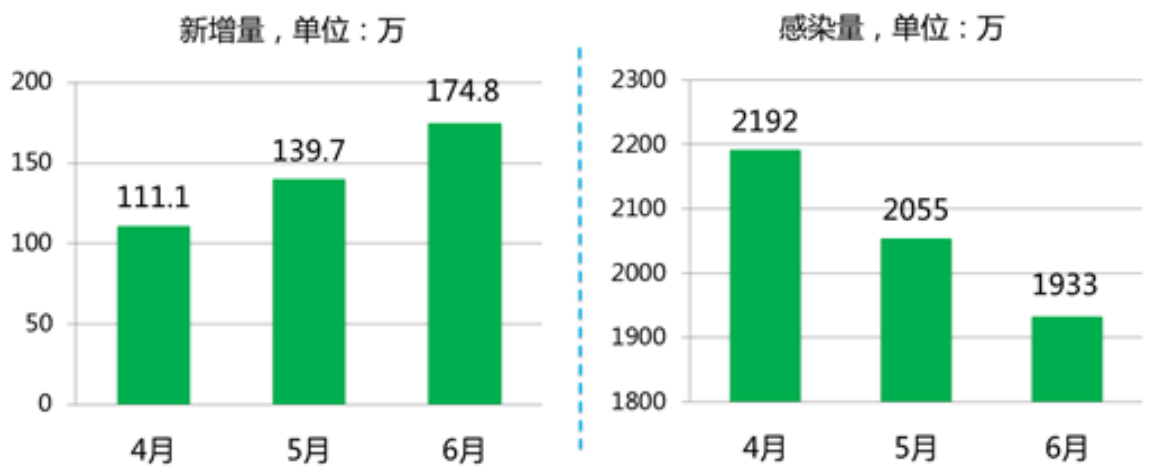

Figure 3. New increment and infection statistics of mobile malicious programs

\section{Conclusion}

In this paper, we aims to design and implement the instruction system based on WeChat. In this paper, this system is discussed in details including its design and implement, at last, its executing efficiency is showed. The exact experimental results indicate that the design objective of this paper is achieved.

\section{Reference}

[1] J. Rissanen, A universal data compression system, IEEE Trans. Inform. Theory, vol. 29, pp. 656-664, Sept. 1983.

[2] J. Rissanen, Strong optimality of the normalized ML models as universal codes and information in data, IEEE Trans. on Information Theory, vol.IT-47, No. 5, pp.1712-1717, 2001.

[3] S.Forchhammer, X.Wu, J.D.Andersen, Optimal context quantization in lossless compression of image data sequences, IEEE Transactions on Image Processing 13(4), pp.509-517, Apr. 2004.

[4] S.Forchhammer, $\mathrm{X}$.Wu, Context quantization by minimum adaptive code length, in: Proc. of IEEE Inter. Symposium on Information Theory, Nice, France, pp.246-250, June 2007.

[5] Min Chen, Jianhua Chen, Affinity propagation for the Context quantization, Advanced Materials Research, Vols. 791, pp.1533-1536, 2013.

[6] Enhancing Teaching and Learning through Education Data Mining and Learning Analytics[J], Education Department of America, pp.336-339, 2012.

[7] Bapler.P\&Murdoch, Academic Analytics on Data Mining in Higher Education. Intemational Jounlal for the Scholarship of Teaching and Leanling, Vol.4(2), pp.1926-1933.2013. 